\title{
An EPR and Crystallographic Investigation of Copper-doped Cadmium Creatinine Sulfate
}

\author{
Michael J. Colaneri ${ }^{1}$, Simon J. Teat ${ }^{2}$ and Jacqueline Vitali ${ }^{3}$ \\ ${ }^{1}$ Department of Chemistry and Physics, SUNY at Westbury, Old Westbury, New York \\ 11568, USA, colanerim@oldwestbury.edu \\ ${ }^{2}$ Advanced Light Source, Lawrence Berkeley National Lab , Berkeley, CA 94720, USA, \\ sjteat@1bl.gov \\ ${ }^{3}$ Department of Physics and Department of Biological, Geological \& Environmental \\ Sciences, Cleveland State University, Cleveland, OH 44115, USA, j.vitali@,csuohio.edu
}

Single crystal Electron Paramagnetic Resonance (EPR) spectroscopic and X-ray diffraction experiments were carried out to determine the crystal structure of cadmium creatinine sulfate and to identify the doped $\mathrm{Cu}(\mathrm{II})$ binding site. Unlike other metal creatinine complex structures, the metal is not in coordination with the creatininium cation, but forms a hexahydrate which is buffered from the creatininium by an intervening sulfate ion. Both the EPR measured $\mathrm{g}(2.44,2.13,2.08)$ and copper hyperfine $(325,77,0 \mathrm{MHz})$ tensors and structure are consistent with doped copper replacing cadmium in the hexahydrate complex. The room temperature EPR tensors were similar to those previously observed for copper hexahydrate in other systems, for example in doped Tutton salts, but only those measured at very low temperatures. In these other systems, vibration couplings stemming from dynamic Jahn-Teller effects cause tensor averaging at temperatures up to room temperature. The reasons why this does not happen in the current host structure is currently being investigated. Progress towards analyzing and correlating the $\mathrm{Cu}((\mathrm{II}) \mathrm{EPR}$ tensor characteristics and the crystal structure will be presented. 